\title{
Intermédialités
}

Histoire et théorie des arts, des lettres et des techniques

Intermediality

History and Theory of the Arts, Literature and Technologies

\section{Programmer un pays}

\section{Cybernétique et matérialisme dialectique en RDA}

\section{Jérôme Segal}

Numéro 13, printemps 2009

Programmer

Programming

URI : https://id.erudit.org/iderudit/044043ar

DOI : https://doi.org/10.7202/044043ar

Aller au sommaire du numéro

\section{Éditeur(s)}

Centre de recherche sur l'intermédialité

ISSN

1705-8546 (imprimé)

1920-3136 (numérique)

Découvrir la revue

Citer cet article

Segal, J. (2009). Programmer un pays : cybernétique et matérialisme dialectique en RDA. Intermédialités / Intermediality, (13), 115-139.

https://doi.org/10.7202/044043ar
Résumé de l'article

Définie comme science générale de la régulation et de la communication s'appliquant aussi bien aux machines, aux êtres vivants qu'à la société, la cybernétique a été profondément marquée aussi bien par son contexte de développement, la Deuxième Guerre mondiale, que par les milieux où elle fut appliquée, à l'époque de la guerre froide. À l'Est, si la cybernétique fut au départ considérée comme une science " bourgeoise ", l'appareil d’État a dû se rendre à l'évidence, son utilisation était nécessaire dans bien des domaines, à commencer par celui des technologies militaires. De son côté, un philosophe influent, Georg Klaus (1912-1974), a su convaincre la direction du parti de la compatibilité de la cybernétique avec le marxisme-léninisme. Dès lors, sous cette double impulsion, les années 1960 ont été marquées par une véritable conversion du pays, la cybernétique devenant la science de la programmation, en informatique bien sûr, mais aussi pour la planification économique et dans bien d'autres domaines.
Ce document est protégé par la loi sur le droit d'auteur. L'utilisation des services d’Érudit (y compris la reproduction) est assujettie à sa politique d'utilisation que vous pouvez consulter en ligne.

https://apropos.erudit.org/fr/usagers/politique-dutilisation/ 


\title{
Programmer un pays
}

\section{Cybernétique et matérialisme dialectique en RDA}

\author{
JÉrôme SEgal
}

Control and Communication in the Animal and the Machine», tel est

le sous-titre du livre le plus célèbre du mathématicien étasunien Norbert Wiener, paru en anglais, à Paris, en 1948. Fruit d'un riche travail interdisciplinaire, Cybernetics décrit une nouvelle théorie générale des systèmes conçue pour pouvoir également s'appliquer à la gouvernance des sociétés. Deux idées sont au centre de la cybernétique: l'universalité des principes de régulation, et la définition de la notion d'information pour caractériser ces phénomènes de régulation ${ }^{1}$.

Développées en même temps que la théorie mathématique de la communication de l'ingénieur Claude Shannon, les applications de la cybernétique font partie de l'essor de ce qu'il est convenu d'appeler la théorie de l'information. Très marquée par son contexte de naissance, la Deuxième Guerre mondiale puis, aussitôt après, la guerre froide, cette théorie permet à l'historien d'étudier avec minutie les liens entre science et idéologie². L'histoire du premier réseau informatique de défense, l'ARPANET (Advanced Research Projects Agency Network), dont l'Internet actuel est issu, trouve ainsi sa place dans l'histoire de la notion d'information, «archéologie» pourrait-on dire, au regard du rôle implicite que joue cette notion. En s'intéressant aux fonctions de la cybernétique en République démocratique allemande (RDA), on parvient à repérer les différentes conditions de possibilité du discours concernant la légitimité de la

1. La genèse de la cybernétique, ainsi que ses différentes applications, sont au centre de Jérôme Segal, Le Zéro et le Un - Histoire de la notion scientifique d'information, Paris, Éditions Syllepse, 2003 (disponible en ligne à livreoı.free.fr, dernière consultation le 8 mars 2010).

2. David A. Mindell, Slava Gerovitch et Jérôme Segal, «From Communications Engineering to Communications Science: Cybernetics and Information Theory in the United States, France, and the Soviet Union », dans Mark Wayne Walker (dir.), Science and Ideology: a Comparative History, Londres et New York, Routledge, 2003, p. 66-95. 
cybernétique. Celles-ci se laissent tout simplement mieux appréhender dans un État hautement centralisé $e^{3}$ De plus, on trouve en RDA, en raison de son aspect dictatorial, la volonté de promouvoir une application «totale» de la cybernétique. Le pays devait être programmé selon des plans adoptés lors des différents congrès où le « programme » du parti était débattu. Une étude des différentes institutions, ainsi que le dépouillement systématique des revues dans lesquelles les controverses évoquées par les témoins de l'époque se retrouvent facilement, permet de nouvelles recherches sur l'histoire de la réception d'une théorie scientifique qui mêle à la fois histoire des sciences, histoire des institutions mais aussi histoire politique et économique ${ }^{4}$. Par ailleurs, ce travail de recherche est représentatif d'une étude historique plus large de la théorie de l'information's.

L'existence d'une position officielle vis-à-vis de la cybernétique, exprimée à travers les différents organes de presse du parti socialiste unifié (SED) et sanctionnée par un ensemble de discours, permet de structurer cette étude de façon chronologique en distinguant trois grandes étapes: la «contamination» par une science bourgeoise, au début des années 1950, la reconnaissance officielle de ces théories en tant que «confirmation du matérialisme dialectique» et, enfin, la période de normalisation.

\section{LA CYBERNÉTIQUe À L’EST DANS LES ANNÉES 1950}

La première trace que l'on retrouve de la cybernétique en RDA est la traduction d'un article paru un mois plus tôt en URSS, en avril 1952, intitulé «Cybernétique, "science" des obscurantistes ${ }^{6} »$. La parution si rapide de cet article témoigne de

3. Nous délaissons sciemment ici le vocable «totalitaire », trop souvent utilisé pour condamner d'un seul souffle l'expérience communiste en l'assimilant aux périodes fascistes.

4. Des entretiens ont été réalisés en 1996 et 1997 avec Klaus Fuchs-Kittowski, Rainer Thiel, Günter Kröber, Heinz Liebscher, Rainer Thiel et Klaus-Dieter Wüstneck.

5. Le présent article est issu de deux publications de l'auteur, rédigées en allemand, «Kybernetik in der DDR - Begegnung mit der marxistischen Ideologie», Dresdner Beiträge zur Geschichte der Technik und der Technikwissenschaften, 27, 2001, p. 47-75 et «Kybernetik in der DDR - dialektische Beziehungen », dans Cybernetics - Kybernetic - The Macy Conferences 1946-1953, Essays \& Dokumente, Claus Pias (dir.), ZürichBerlin, diaphanes, 2004, p. 227-251. Depuis, un livre collectif a été consacré à ce sujet, Frank Dittmann et Rudolf Seising, Kybernetik steckt den Osten an. Aufstieg und Schwierigkeiten einer interdisziplinären Wissenschaft in der DDR, Berlin, trafo, 2007.

6. M. Jaroschevski, «Die Kybernetik - eine neue "Wissenschaft" der Obskuranten ", Neue Welt, 7, 1952, Heft 10, p. 1193-1195. On trouve de même en URSS, en 1953, un article 
l'influence en RDA de la campagne lancée par quelques philosophes soviétiques contre la cybernétique, et il convient dès lors de suivre l'évolution de la ligne officielle définissant le statut de la cybernétique.

Dès la fin de la guerre, en 1946, à la suite du rapport de l'académicien V. Kovalenkov, un plan quinquennal avait été mis en place pour mettre en œuvre à grande échelle l'automatisation de l'industrie. Le journaliste marxiste Jacques Bergier en avait fait état dans un article intitulé «Un plan général d'automatisation des industries », publié dans Les Lettres françaises le 15 avril 1948. Larticle portait comme sous-titre «Nés des poupées automates, des mathématiques pures et de la physique corpusculaire, les robots prendront bientôt la relève des travailleurs ». Malgré la mention de cette menace pesant sur la classe ouvrière, le ton général de l'article n'était pas très critique. Bergier notait qu'on assistait à « une révolution plus importante encore que celle de l'âge atomique (...)».

En URSS, à partir de 1952, alors que la cybernétique connaît un grand succès dans les pays «impérialistes », une série d’articles paraissent dans diverses revues spécialisées de sciences humaines, dénonçant la cybernétique comme «pseudo-science». D’un côté, les scientifiques avaient la possibilité de travailler sur des sujets inclus dans la cybernétique, mais sans utiliser le mot, en partant le plus souvent de la théorie mathématique de la communication. D’un autre côté, la cybernétique était toujours évoquée de manière péjorative et jusqu'en 1955, le célèbre Petit dictionnaire de philosophie comportait un article très critique à ce sujet.

Il y a plusieurs explications à cette attitude plus que réservée des marxistes: il s'agissait d'abord d'une théorie "made in USA», et par conséquent presque condamnée d'avance en période de guerre froide. Ensuite, du point de vue des présupposés philosophiques, la cybernétique comportait des conceptions idéalistes liées au rôle prépondérant accordé au raisonnement analogique qui allaient à l'encontre du matérialisme dialectique ${ }^{7}$. De plus, la cybernétique

signé du pseudonyme "Le Matérialiste» dans la revue Voprosy filosofii (5, 1953, p. 210219), intitulé «À qui sert la cybernétique?», dont une traduction paraît en RDA: Der Materialist, «Wem dient die Kybernetik? ", Sowjetwissenschaft - Naturwissenschaftliche Abteilung, 4, 1956, p. 302-309.

7. Dans Cybernetics, Wiener écrivait: «L'information est de l'information, pas de la matière ni de l'énergie. Aucun matérialisme qui ne reconnaît pas ce fait ne peut survivre aujourd'hui ", ce qui constituait un défi pour les philosophes marxistes. Norbert Wiener, Cybernetics or Control and Communication in the Animal and the Machine, Paris, Cambridge (Mass.) et New York, Hermann \& Cie Éditeurs, The Technology Press et John Wiley \& Sons Inc., 1948, p. 155. 
représentait un certain danger pour plusieurs disciplines : en économie, la cybernétique pouvait, avec sa théorie des systèmes autorégulés, justifier le système capitaliste, tel qu'il était vulgarisé par des règles comme «l'offre et la demande déterminent le marché». Enfin, de nombreux écrits des premiers cybernéticiens ou théoriciens de l'information amenaient à considérer l'argent comme une valeur essentielle. Le fait que l'information soit présentée comme une nouvelle grandeur conduisit quelques scientifiques à comparer l'information à la monnaie, ou même à exprimer l'information... en dollars.

Le changement s'opère en URSS au milieu des années 1950, la cybernétique quittant son statut de science «impérialiste» pour devenir digne d'intérêt. Wiener n'est alors plus considéré comme un «scientifique impérialiste », grâce à ses écrits très critiques sur la société américaine, notamment la deuxième édition de The Human Use of Human Being où il s'en prend au maccarthysme ${ }^{8}$. Il a, de plus, reconnu l'importance des travaux soviétiques (de Kolmogorov notamment), et milite contre l'utilisation de l'arme atomique. Avec la cybernétique, il entend développer de nouvelles prothèses et créer des procédés de traduction automatique. Son amitié avec le généticien britannique marxiste J. B. S. Haldane est également parfois mise de l'avant. En outre, la cybernétique est devenue nécessaire pour les militaires: un rapport confidentiel rédigé par le vice-amiral Aksel Berg semble jouer de ce point de vue un rôle décisif, tout comme la publication par trois scientifiques travaillant à titres divers pour l'armée, Sobolev, Lyapunov et Kitov, d'un article ouvertement favorable aux théories cybernétiques. Enfin, dans le domaine public, c'est sans aucun doute l'exposé produit par Arnost Kolman, le 19 novembre 1954 devant l'Académie des sciences sociales du Comité central du parti communiste soviétique, qui marque ce changement d'attitude de la part de la communauté scientifique soviétique. Son intervention a pour titre «Qu'est-ce que la cybernétique?» et sera publiée, une première fois, quelques mois plus tard en RDA. Dans son allocution, Kolman commence ainsi son argumentation:

[...] il paraît erroné d'imaginer que nos adversaires s'adonnent à des préoccupations dépourvues de sens, qu'ils y consacrent des moyens énormes, créent des instituts

8. Norbert Wiener, The Human Use of Human Beings: Cybernetics and Society, Boston, Houghton Mifflin, 1950. Cybernetics fut ainsi relu, et on découvrit des passages où Wiener écrit par exemple: «In a society like ours, avowedly based on buying and selling, in which all natural and human resources are regarded as the absolute property of the first business man enterprising enough to exploit them, these secondary aspects of the means of communication tend to encroach further and further on the primary ones », p. 189. 
entiers, organisent des conférences nationales et des congrès internationaux, éditent des revues spéciales -, tout cela uniquement pour discréditer l'enseignement de Pavlov et introduire l'idéalisme et la métaphysique dans la psychologie et la sociologie. La propagande idéaliste et l'agitation belliciste disposent de moyens beaucoup plus efficaces et moins coûteux que de s'occuper de cybernétique. D’autre part, on n'a pas le droit de sous-estimer le fait que l'un des plus grands physiciens de notre temps, Louis de Broglie, a présidé un colloque sur la cybernétique en France en $1951^{9}$.

Sans analyser en détail la suite de l'article de Kolman, notons qu'il consacre une partie importante de son exposé à la «théorie mathématique de l'information ».

Il semblerait que cette prise de position relativement courageuse ait valu à Kolman son transfert à Prague et des réformateurs est-allemands, comme le célèbre Robert Havemann, s'en sont par la suite émus ${ }^{10}$. En URSS, notons encore le rôle joué par la parution en 1958 non seulement de la version russe du livre emblématique de Wiener, mais aussi du premier livre à grande diffusion sur la cybernétique, même s'il est discrètement intitulé Signal ${ }^{11}$. Il est édité en RDA sous le titre Kybernetik en 1962. Comme le note Heinrich Kindler, on assiste à partir de ce moment-là à une avalanche de titres et publications sur la cybernétique «comme des champignons qui sortent de terre ${ }^{12}$ ». Qu'en était-il en RDA?

L'histoire de la cybernétique en RDA, telle qu'on peut l'aborder grâce aux documents d'archives et aux entretiens avec les témoins de l'époque, est plus «dense» et ne se laisse pas schématiser par de simples relations causales. Si la période de rejet de la cybernétique est fortement calquée sur l'attitude des scientifiques soviétiques, son introduction en des termes plus positifs résulte de diverses influences, à la fois soviétiques avec, en particulier, la publication de

9. Arnost Kolman, «Was ist Kybernetik? », Forum - Wissenschaftliche Beilage, 9, 1955, $\mathrm{n}^{\circ}$ 23. L'original du même titre, en russe, a paru dans Voprossy Filosofii, 4, 1955, et pour la traduction française citée ici, voir «La cybernétique vue par un philosophe soviétique», La Pensée, 68, 1956, p. 14-34.

10. Robert Havemann, Dialektik ohne Dogma, Hambourg Reinbeck, 1964, p. 15. Le physicien qui est exclu cette année-là du parti communiste est-allemand écrit à propos de Kolman: «L'histoire des difficultés de sa vie est une preuve sans fin de ce que j’ai dit. »

11. Igor Andreevich Poletajew, Kybernetik, kurze Einführung in eine neue Wissenschaft, Berlin, VEB Deutscher Verlag der Wissenschaften, 1962.

12. Heinrich Kindler, «Mathematische und physikalisch-technische Probleme der Kybernetik», Universitätszeitung TU-Dresden, $1^{\text {er }}$ avril 1962, p. 3. 
l'exposé de Kolman, mais aussi propres à la RDA, avec la recension des livres et publications parus à l'Ouest.

La première mention de la cybernétique trouvée dans cette revue figure dans la recension par Hans Fortner du livre de Louis Couffignal, Les machines à penser. Fortner fait allusion à « un domaine qui est considéré dans certains pays comme une branche de la science (Wissenschaftszweig): la "cybernétique ${ }^{13 "}$ ". Les Allemands de l'Est n'étaient pas seulement tournés vers ce qui se passait à l'Est, et c'est ce qui fait une des spécificités de l'histoire de la réception de la cybernétique dans ce pays.

Selon tous les témoins interrogés, c'est à un philosophe que l'on doit principalement l'introduction de la cybernétique en RDA, Georg Klaus (19121974). Issu d'un milieu modeste, Klaus put bénéficier d'un soutien exceptionnel de la ville de Nuremberg en raison des facilités dont il faisait preuve à l'école. Il commence à étudier les mathématiques à l'Université d'Erlangen et ses activités politiques (direction du parti communiste en Bavière du Nord) le font condamner par les nazis en 1933. Après différents internements, il finit par être déporté à Dachau et survit sur le plan intellectuel grâce aux parties d'échecs qu'il joue à l'aveugle, comme le héros du Joueur d'échec de Stefan Zweig ${ }^{14}$. Il semble que ce soit cette expérience qui l'ait amené, après-guerre, à se tourner vers la logique formelle qui, avant même la cybernétique, avait également été dépréciée comme «science bourgeoise ».

Klaus fait partie des quelques grands professeurs qui n'ont pas séjourné en URSS et qui ne parlent pas du tout russe. C'est toutefois lui qui fera publier dans Forum l'exposé de Kolman, tandis qu'un de ses deux proches élèves, Rainer Thiel, estime que Klaus a dû lire le livre de Wiener en 1952 (il lisait parfaitement le français et l'anglais). À propos de la publication en allemand du texte de Kolman (publié, donc, à l'initiative de Klaus), le médecin et physiologiste Hans Drischel écrit dès 1963: «ainsi la glace fut brisée ${ }^{15}$ ».

La première publication d'un Allemand de l'Est sur la cybernétique (outre la recension citée ci-dessus) correspond à une communication donnée par

13. Hans Fortner, «Louis Couffignal: Denkmaschinen», Deutsche Zeitschrift für Philosophie, 4, 1956, Heft 3, p. 371-375.

14. Klaus témoigne de ce passage douloureux de sa vie dans son texte «Erlebte Schachnovelle», dans Anita Karau et Wenzel Renner (dir.), Schwarz und Weiss, heitere und ernste Begegnungen mit dem königlichen Spiel, Berlin, 1960, p. 164-182.

15. Hans Drischel, "Medizin, Biologie und Kybernetik", dans Kybernetik in Wissenschaft, Technik und Wirtschaft der DDR, Berlin, Akademie Verlag, 1963, p. 154184, ici, p. 155. 
Klaus en 1957, sortie sous forme de livre: Cerveau électronique contre cerveau humain? (sur les problèmes philosophiques et sociaux de la cybernétique). Comme le fait remarquer le philosophe Heinz Liebscher, cet exposé est l'occasion pour le philosophe de se prononcer à la fois «contre une vulgarisation matérialiste mécaniste de la cybernétique et contre le refus des pseudo-matérialistes dialectiques $^{16}$ ». La cybernétique est ici présentée comme une théorie des nouveaux calculateurs, et comme dans l'exposé de Kolman, son importance est comparée à celle de l'énergie atomique.

Un point de repère important dans l'introduction de la cybernétique en RDA est le premier article paru en 1958 dans la revue Einheit, évidemment très liée au pouvoir politique, puisque éditée par le comité central du SED. À la suite de cet article, la cybernétique est déjà officieusement acceptée. Dans l'article «Au sujet de quelques problèmes liés à la cybernétique », Klaus développe une véritable stratégie pour convaincre les «camarades» de la revue. Ce n'est qu'après sept pages sur Marx et l'URSS que le mot cybernétique apparaît, et ce, en référence à la question concernant la possibilité, pour une machine, de «penser ». Il rappelle ainsi que sans l'ENIAC ${ }^{17}$, les Américains n'auraient pas pu disposer de bombe atomique, et ses exemples sont loin d'être choisis au hasard lorsqu'il compare différentes machines: l'ENIAC accomplit 250 multiplications par seconde, le BESK à Stockholm, 3000, et enfin le BESM à Moscou, 8000! De plus il utilise «l'effet Spoutnik» avant d'introduire le mot cybernétique. Pas de Spoutnik sans calculateurs ${ }^{18}$ ! Il précise, de plus, que ces nouvelles machines sont caractéristiques de la «révolution scientifique et technique alors en marche dans le Bloc de l'Est».

Au sujet de l'expression «machines pensantes», il écrit que des arguments inédits apparaissent dans le débat avec «une nouvelle théorie mathématique connue sous le nom de théorie de l'information ». Il propose, d'ailleurs, au lecteur une reproduction du schéma de base d'une communication selon Shannon, et ajoute: «La théorie de l'information montre seulement que l'on peut définir

16. Heinz Liebscher (dir.), Georg Klaus - zu philosophischen Problemen der Einzelwissenschaften, Berlin, Akademie Verlag, 1978, p. 9.

17. «Electronic Numerator Integrator and Computer», construit en 1945 aux ÉtatsUnis.

18. À propos du Spoutnik, on peut rappeler les remises en question provoquées outreAtlantique par ce succès soviétique. Un éditorial des IRE - Transactions on Information Theory du 3 décembre 1957 porte pour titre «Sputnik etc. » et s'attache à définir quelques priorités entre science fondamentale et appliquée, s'inquiétant du caractère orienté vers les profits de la recherche américaine. 
mathématiquement la notion d'information. Il y a une analogie saisissante avec la thermodynamique. Le contenu informationnel se trouve formellement égal à l'entropie ${ }^{19}$. Et dans la troisième partie de sa publication, il affirme que «l'analogie entre homme, animal et machine conduit à la création du concept de cybernétique». Un seul article dans Einheit ne suffit toutefois pas à vaincre toute résistance et légitimer la discipline. On retrouve encore au début des années 1960 de sévères critiques de la cybernétique, de la part d'autres philosophes surtout, mais aussi de scientifiques qui ne voulaient pas être mêlés à ces questions philosophiques.

\section{CONTROVERSES EN RDA SUR LA CyBERnÉtIQUE (1961-1962)?}

122 Pourquoi ce point d'interrogation? S'il y a bien eu des débats et conflits entre institutions ou scientifiques, ceux-ci ne sont que très peu apparents dans les revues ou monographies. À partir du moment où il devient clair que la cybernétique est officiellement encouragée, seuls quelques scientifiques osent encore la remettre en cause. De plus, des problèmes de personnes viennent se greffer sur ces polémiques. Par exemple, le philosophe Hermann Ley, qui était un ennemi irréductible de Klaus, s'oppose systématiquement à la cybernétique, et publie, en 1961, un livre montrant comment les idées de Wiener ont «tout de l'idéologie bourgeoise», en particulier la partie qui concerne la définition de l'information ${ }^{20}$.

On peut, toutefois, distinguer à nouveau quatre types d'arguments différents jouant en faveur de l'adoption progressive des théories cybernétiques: le rôle de Khrouchtchev, le travail fourni par Klaus, la situation politique et l'importance de la littérature étrangère, enfin, les différents succès techniques liés de façon plus ou moins explicite à la cybernétique.

Dans le cadre de l'automatisation, Khrouchtchev avait déjà appelé, en 1956, lors du $20^{\mathrm{e}}$ congrès, à son application impérative dans l'industrie, créant d'ailleurs à cet effet un «ministère pour l'Automation ». Le colloque sur l'automation, qui se tient en 1956, marque à sa façon l'acceptation des théories cybernétiques,

19. Georg Klaus, «Zu einigen Problemen der Kybernetik», Einheit, 13, n 7, juillet 1958, p. 1026-1040, ici, p. 1032 et 1033.

20. «Le fétiche de Wiener est l'information technique. (...) Les lois sociales, les classes et les rapports de propriété ont [cependant] une réalité matérielle qui évolue au cours de l'activité de production et dans la lutte des classes et pas par la médiation d'information.» Hermann Ley, Dämon Technik?, VEB Deutscher Verlag der Wissenschaften, Berlin, 1961, p. 69 et 73 . 
même si beaucoup ne se risquent pas encore à utiliser le néologisme forgé par Wiener (qui ignorait qu’Ampère l'avait utilisé en 1834). Le Conseil scientifique pour la cybernétique, dont la direction est confiée à Berg, est créé à l'Académie des Sciences soviétique en avril 1958. Dans le domaine de l'automation, l’URSS acquiert d'ailleurs bientôt un rang important sur la scène internationale, grâce à l'organisation de la première conférence de la Fédération internationale pour la commande automatique. Fort du fait que l'automation devient dès lors admise, Berg prend la parole à l'Académie en 1961 et annonce :

La cybernétique est une nouvelle science qui est de plus en plus utilisée. Elle aspire à l'étude des processus de commande apparaissant dans la nature, l'industrie et la société humaine, c'est-à-dire à couvrir pratiquement toute l'activité humaine. Pour cette raison nous exigeons que la base idéologique sur laquelle est développée cette science en URSS soit impeccable ${ }^{21}$.

C'est en cette même année 1961, lors du $22^{\mathrm{e}}$ congrès, que la cybernétique est explicitement mentionnée par Khrouchtchev: «Il est impératif d'organiser de larges applications de la cybernétique, du calcul électronique et du contrôle des installations dans la production, la recherche, la conception et le design, la

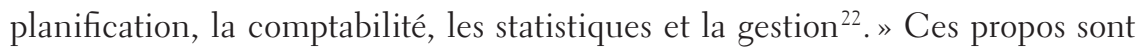
aussitôt mis en application en RDA un mois plus tard, en novembre 1961, lors du $14^{\mathrm{e}}$ Plenum du Comité central du SED. La question à l'ordre du jour est la conception de la science comme moyen d'aide directe pour l'amélioration de la production et surtout de la productivité. La cybernétique prend alors une place de premier plan dans la révision des objectifs du plan septennal 1959-1965.

Si la politique scientifique de la RDA est en partie déterminée par les événements soviétiques, elle possède ici aussi une dynamique propre qu'il convient de ne pas négliger. Klaus organise en avril $1961 \mathrm{avec}$ la revue Einheit une journée d'information [wissenschaftliche Beratung] sur le thème «Cybernétique, philosophie et société ». Thiel indique dans son rapport que cette

21. Nous soulignons et traduisons la version anglaise produite par Loren R. Graham "Cybernetics in the Soviet Union », Survey: a Journal of Soviet and East European Studies, $\mathrm{n}^{\circ} 52$, juillet 1964 , p. 3-18, ici, p. 3.

22. Voir Georg Klaus, «Für und Wider die Kybernetik - eine Betrachtung zum XXII. Parteitag», Deutsche Zeitschrift für Philosophie, 10, 1962, Heft 5, p. 582-601, entièrement consacré au $22^{\mathrm{e}}$ Congrès du PCUS et intitulé " Pour ou contre la cybernétique ». Ce Congrès a donné lieu en 1961 en RDA à deux publications de Chruschtschow (Khrouchtchev en français): Der Triumph des Kommunismus ist gewiß et Programm und Statut der KPdSU. 
réunion a rassemblé plus de trente scientifiques d'horizons différents, marquant une «rupture» dans l'histoire de la cybernétique en $\mathrm{RDA}^{23}$.

Le premier livre est-allemand sur la cybernétique faisant date sort en cette même année 1961, au mois d'août. Il s'agit de La cybernétique d'un point de vue philosophique, du même Klaus ${ }^{24}$. Après deux ans passés à la direction du groupe de travail de philosophie à l'Académie des Sciences, Klaus est de plus devenu, cette année-là, «membre ordinaire » de l'Académie. Il entend montrer dans son livre que non seulement la cybernétique est compatible avec le matérialisme dialectique, mais encore qu'elle en confirme la validité. Un article paru le 15 octobre 1960 dans Neues Deutschland (le quotidien du parti), avec un titre sans le moindre ton polémique, «Boucles de contrôle et organismes », comportait d'ailleurs déjà une section intitulée «confirmation du matérialisme dialectique ${ }^{25} »$. Il prenait cependant soin d'intituler une section suivante de l'article «les robots ne peuvent pas penser dialectiquement». La cybernétique sert la philosophie, mais ne saurait en aucun cas s'y substituer. On assiste peu à peu, dans le travail de Klaus, à un phénomène de réappropriation de la cybernétique par la philosophie marxiste, dont l'ampleur mérite d'être évoquée.

À grand renfort de citations «politiquement correctes», Klaus montre d'abord que l'idée de régulation est profondément ancrée dans le matérialisme dialectique ${ }^{26}$. Ainsi dans L'idéologie allemande, Marx et Engels n'appelaient-ils pas le prolétariat à s'approprier les moyens de production et parvenir ainsi à une «auto-commande »? À propos du Capital, Klaus montre de plus comment le rapport entre le flux de capital et la détermination du prix est exprimé par

23. Rainer Thiel, «Kybernetik - Philosophie - Gesellschaft», Einheit, 16, Beilage zu Heft 7, juillet 1961, p. 1-32. Interrogé sur cet événement, il précise qu’il s’agissait « d’une certaine rupture».

24. Georg Klaus, Kybernetik in philosophischer Sicht, Berlin, Dietz Verlag, 1961.

25. Georg Klaus, «Regelkreise und Organismen », Neues Deutschland, 15, 1960.

26. Il semble, d'après le nombre de citations, que l'ouvrage de Lénine Matérialisme et empiriocriticisme (1908) ait joué un grand rôle dans le développement de ces idées, poursuivant le parallèle entre physique moderne et cybernétique. Parmi les douze thèses défendues par Klaus lors de la journée d'information, la sixième est entièrement consacrée à l'interprétation de ce livre de Lénine selon lequel «la science moderne est sur le point d'engendrer le matérialisme dialectique». Les documents d'archives relatifs à cette «wissenschaftliche Beratung» figurent aux Archives nationales, Stiftung Archiv der Parteien und Massenorganisationen der DDR, sous la cote DY30 IV 2/9.10/5. On trouve dans ce fonds d'archives un texte de dix pages concernant les thèses que Klaus fait approuver à cette occasion ainsi que la correspondance alors adressée à la revue Einheit. 
Marx en des termes "pré-cybernétiques ». Cherchant à illustrer de manière pédagogique son interprétation, il reprend dans son livre ce schéma qu'il avait déjà donné dans une publication de $1960^{27}$ (fig. 1).

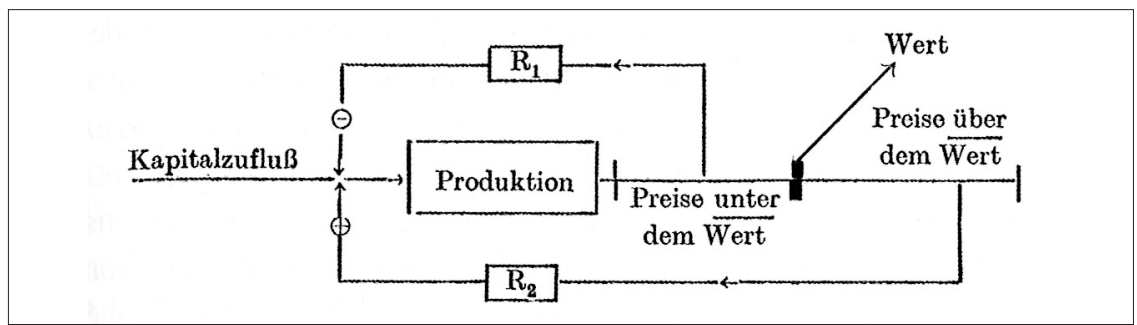

Fig. 1: Georg Klaus, Deutsche Zeitschrift für Philosophie, 8, 1960, p. 1277. Traduction (de gauche à droite et de haut en bas): apport de capitaux; production; prix au-dessous de la valeur; valeur; prix au-dessus de la valeur.

Le philosophe est-allemand finit par proposer une série d'équivalences entre la boucle de contrôle et l'unité dialectique, le rétrocontrôle et le rapport dialectique, entre cause et effet, ou encore entre l'information et les rapports particuliers qui unissent matière et conscience. Il distingue par ailleurs quatre aspects dans la cybernétique: régulations, approche systémique, théorie de l'information et théorie des jeux, n'hésitant pas à affirmer que ce dernier aspect permet de modéliser la lutte des classes.

À l'aide d'une analyse cybernétique, il dénonce la loi d'airain des salaires de Lassale, représentée par un «mauvais feedback» (fig. 2).

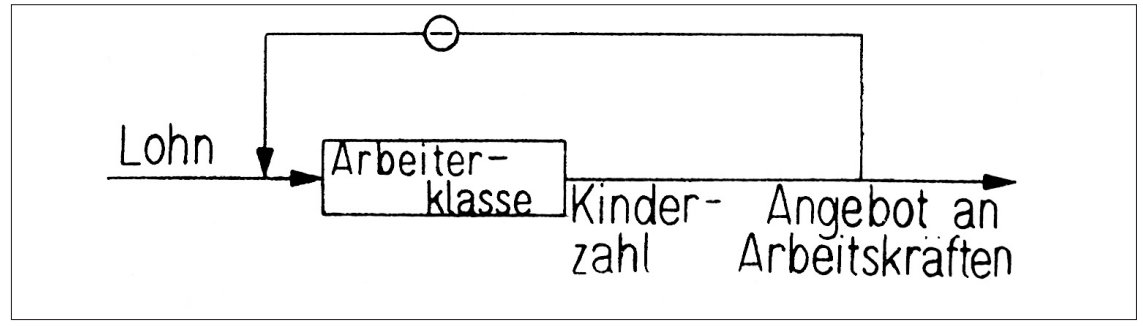

Fig. 2: Georg Klaus, Kybernetik in philosophischer Sicht, Berlin, Dietz Verlag, 1961, p. 489. Traduction (de gauche à droite): salaire; classe ouvrière; nombre d'enfants; offre en force de travail.

27. Dans cette publication, il allait jusqu'à écrire: «Karl Marx a utilisé le premier de façon implicite et par anticipation des modes de pensée de caractère cybernétique, ou - exprimé sous une forme extrême - Karl Marx était le premier cybernéticien!", Deutsche Zeitschrift für Philosophie, 8, 1960, p. 1266-1279, ici p. 1277. 
D’un point de vue méthodique, la cybernétique étend et approfondit le matérialisme dialectique grâce à la méthode de la boîte noire, à l'analogie cybernétique ( «imitation mathématique de la contradiction dialectique ») et à la méthode «trial-and-error». La cybernétique et le marxisme-léninisme finissent par être si étroitement reliés que l'idée apparaît selon laquelle le cybernéticien «bourgeois », tel Monsieur Jourdain la prose, développe le matérialisme dialectique sans le savoir.

Grâce à la cybernétique, ce sont des notions comme l'enthousiasme du travailleur qui apparaissent (fig. 3).

Le marxisme théorique se retrouve enrichi de ces nouveaux écrits. Un livre entier sera ainsi consacré, en 1969, par un marxiste d'Allemagne de l'Ouest, à la théorie du reflet chez Marx dans ses rapports avec la cybernétique ${ }^{28}$. À la place de l'opposition classique entre matière et énergie, Klaus introduit les concepts de fonction et structure. C'est dans ce cadre qu'il propose une interprétation cybernétique et plutôt progressiste du centralisme démocratique, dans laquelle non seulement il remet implicitement en cause le rôle directeur du parti, mais encore il assimile le parti à un système doué des facultés d'apprentissage.

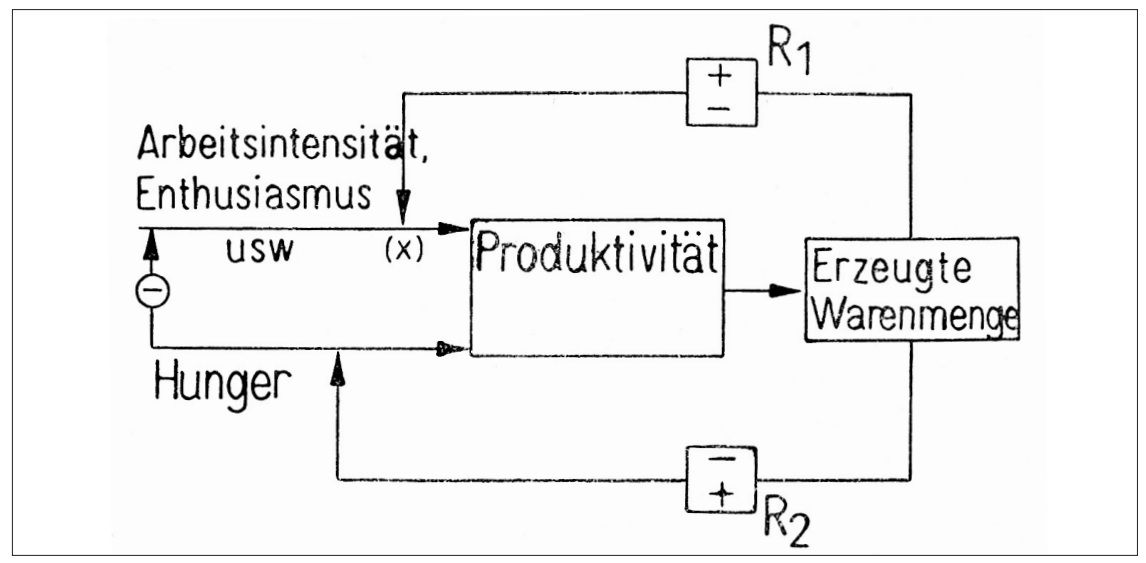

Fig. 3: Georg Klaus, Kybernetik in philosophischer Sicht, Berlin, Dietz Verlag, 1961, p. 509. Traduction (de haut en bas et de droite à gauche): intensité du travail, enthousiasme; faim; productivité; quantité de biens produits.

28. Peter Kirschenmann, Kybernetik, Information, Widerspiegelung. Darstellung einiger philosophischer Probleme im Dialektischen Materialismus, Munich, Verlag Anton Pustet, 1969. 
En ce qui concerne la question du déterminisme ou de l'indéterminisme, la cybernétique apporte également un nouveau point de vue. Celle-ci montre que malgré le «bruit» et les perturbations extérieures, les boucles de contrôle assurent la stabilité du système, et par conséquent, la conception déterministe sous-jacente dans le matérialisme dialectique se trouve renforcée. Encore en 1976, une thèse de doctorat sera consacrée à l'Ouest à l'œuvre de Klaus sur le thème Dialectique et Cybernétique en $\mathrm{RDA}^{29}$.

Au début des années 1960, la cybernétique est également utilisée dans les "pays frères» pour justifier l'économie planifiée. Dans le livre du Polonais Henryk Greniewski qui connut une diffusion internationale impressionnante, à l'Est comme à l'Ouest, La cybernétique sans mathématiques (aussitôt traduit en anglais), on trouve le schéma ci-joint (fig. 4).

La représentation graphique est en effet un moyen de rendre très facilement accessible - de façon immédiatement visuelle et sans nécessiter de connaissances préalables en mathématiques - la structure d'une modélisation. Rendue possible par le cœur même de la théorie cybernétique, avec l'utilisation des notions de rétrocontrôle et de boucles informationnelles, cette modélisation participe d'un désir d'unité du savoir. De nouvelles méthodes de représentations, comme celle de l'ordinogramme ou l'analyse entrée/sortie par diagramme de flux, connaissent dans ce cadre une grande diffusion.

Ces recherches théoriques, axées sur l'interprétation cybernétique du marxisme autant que sur une lecture marxiste de la cybernétique, ont suscité quelques controverses - on ne retrouve que rarement la trace dans les sources imprimées -, et parmi les scientifiques et du côté des représentants «dogmatiques» des sciences humaines.

Connus sous le nom de «Gewis» (pour Gesellschaftswissenschaftler), ces derniers font souvent partie de ces philosophes ou autres qui ont pu bénéficier après la guerre de postes importants dans différentes institutions officielles, alors que leur niveau de formation avait souffert de la guerre, profitant dans ces années de guerre froide du slogan «là où nous ne sommes pas présents, l'ennemi parle à notre place». Il semblerait ainsi que les «Gewis» aient pu noyauter l'école du parti pour y défendre une conception assez dogmatique, pour ne pas dire

29. Gerda Haufe, Dialektik und Kybernetik in der DDR, Berlin, Dunker und Humblot, 1980 (Beiträge zur polit. Wiss. 38 - Doktorarbeit Ruhr-Universität Bochum 1976). L'ouvrage se situe dans une approche exclusivement philosophique et non historique, les citations de Klaus et Marx constituant d'ailleurs, à part égale, la majeure partie de l'ouvrage. 
Model of a centrally planned national economy

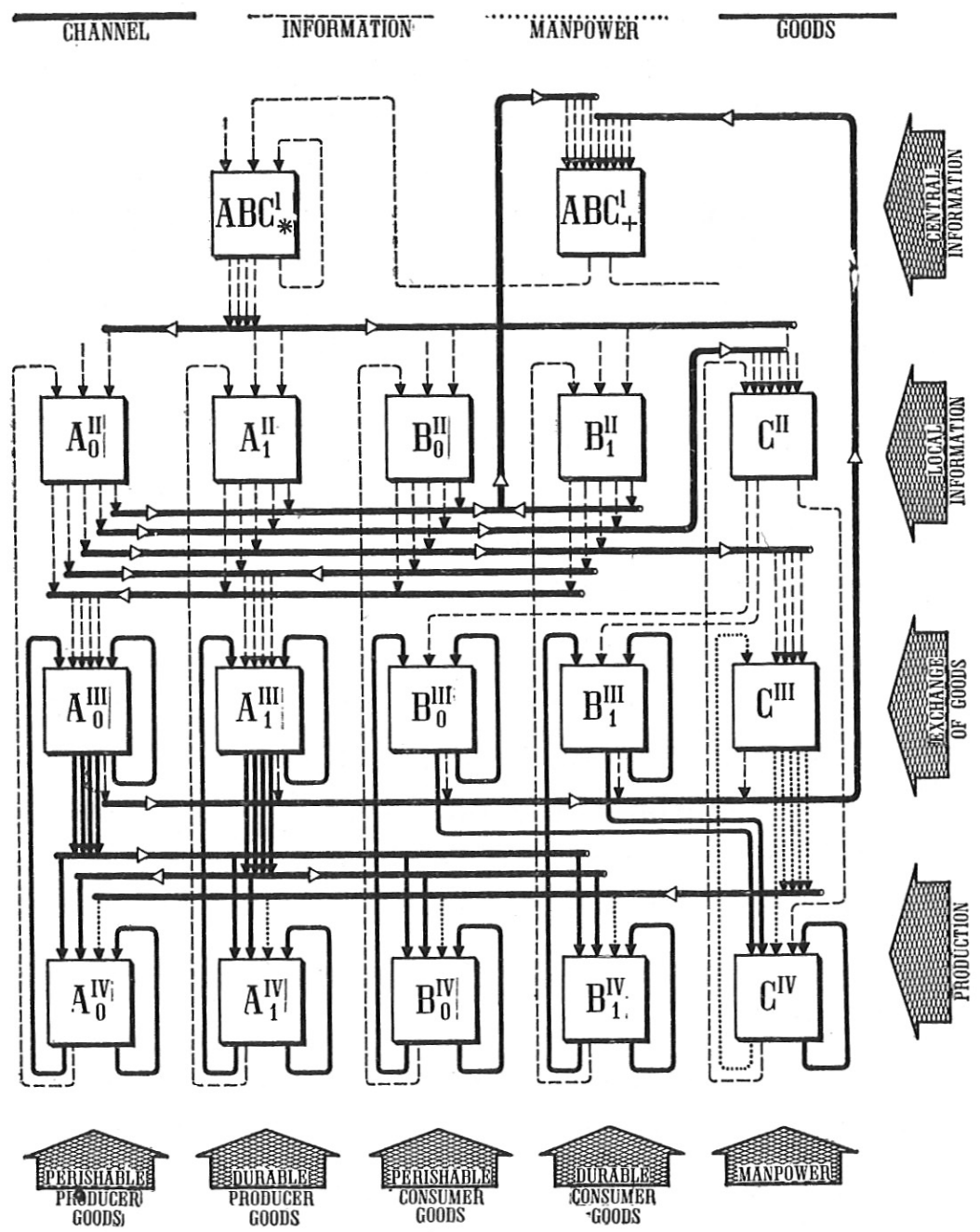

Fig. 4: Henryk Greniewski, Cybernetics without Mathematics, Varsovie, Pergamon Press, 1960, p. 196. 
stalinienne, du marxisme. Dès le rapport de Thiel précédemment évoqué, celuici se plaignait des «conceptions dogmatiques de plusieurs représentants des sciences humaines, ainsi que des rédactions de quelques revues qui avaient gêné [leur] initiative $[. .$.$] » et portaient la responsabilité du « retard de la RDA» dans$ ce domaine.

Du côté des philosophes ou des scientifiques, il y eut aussi des critiques assez fortes, mais le charisme de Klaus, titulaire du prix national de la science et rescapé des camps de la mort, ne permettait pas qu'on puisse s'attaquer à lui dans les revues. Klaus Fuchs-Kittowski a ainsi écrit une recension pour la Deutsche Zeitschrift für Philosophie du principal livre de Klaus (Kybernetik in philosophischer Sicht) qui n'a pas été publiée. Fuchs-Kittowski reprochait au philosophe un certain dogmatisme dans sa présentation de la cybernétique, comme l'annonçait déjà le titre de la recension : «Une contribution au dépassement du dogmatisme dans la philosophie». Il s'en prenait également aux «Gewis». Il est fort probable que la cybernétique, telle qu'elle était présentée par Klaus, finissait par avoir trop de pouvoir, notamment quant à la légitimation de certains domaines des sciences humaines, telle la psychologie encore mal implantée en RDA. Selon une conception relativement scientiste, la cybernétique avait, par exemple, permis de définir en psychologie des notions comme celle de productivité du travail.

Sur le plan scientifique, quelques personnalités importantes restent hostiles à la cybernétique, comme Jacob Segal, résistant juif de la première heure pendant la Deuxième guerre mondiale, ancien déporté et directeur, dans les années 1950, de l'Institut de biologie générale à l'Université Humboldt de Berlin ${ }^{30}$. En 1958, il contestait à la cybernétique toute emprise éventuelle sur des disciplines autres que celles relevant de la technique, et rappelait, au sujet d'une application possible en biologie, qu'on se devait d'être extrêmement vigilant $^{31}$. Quatre ans plus tard, il critique presque chaque point avancé par les cybernéticiens et, à propos des travaux de Shannon et ses épigones, écrit que

30. Là encore, des études biographiques montrent que les intellectuels est-allemands nés bien avant la guerre vivent dans une double culture, intéressés par les travaux soviétiques pour des raisons politiques, et formés par des recherches menées sur les travaux français ou anglais pour des raisons historiques (nous nous référons à un entretien du 11 novembre 1996 avec sa femme, Lilli Segal (1913-1999), également rescapée des camps de la mort, ainsi qu'à son autobiographie, Lilli Segal, Vom Widerspruch zum Widerstand, Essen, Verlag Neuer Weg, 1986).

31. Jacob Segal, Die dialektische Methode in der Biologie, Berlin, Dietz Verlag, 1958, p. 229 . 
« la théorie de l'information a d'abord été développée par Kotelnikov, Kolmogorov et d'autres, en tant que théorie purement mathématique, indépendante de la cybernétique et développée avant elle $^{32} »$. Ainsi la notion d'information se retrouve-t-elle au centre d'une polémique qui cependant, n'est que rarement mentionnée dans les sources imprimées.

Après l'influence des prises de position politiques et le rôle de Klaus, la littérature étrangère introduite en RDA mérite également d'être brièvement évoquée. En dehors de Norbert Wiener, dont la deuxième édition de The Human Use of Human Being semble appréciée, le Britannique W. Ross Ashby bénéficie également des faveurs des scientifiques est-allemands. On retrouve ainsi souvent les notions d'homéostat ou de multistabilité définies dans Design for a Brain ${ }^{33}$ et An Introduction to Cybernetics ${ }^{34}$. De même, le livre de Léon Brillouin, Science and Information Theory (traduit en russe en 1960) fournit des références aux côtés d'autres écrits allemands, cette fois-ci de République fédérale, dont plusieurs témoins reconnaissent aujourd'hui l'importance.

Sur le plan international, le relatif isolement dans lequel entre la RDA après la construction du mur $(1961)$ a également des conséquences. Ainsi, dans le journal de l'Université de Dresde, le directeur de l'institut pour les techniques de régulations, Kindler, appelle les scientifiques de différentes disciplines à s'unir autour des théories cybernétiques, tout comme les partis politiques avaient pu le faire lors de la création de la RDA avec le Nationale Front ${ }^{35}$. Cette métaphore politique est d'autant plus significative qu'il y a bien, à travers l'expansion de la cybernétique, une volonté de retrouver une certaine unité du savoir.

Une fois la RDA isolée, les Gewis perdent leur raison d'être et, sous Ulbricht, toute leur importance. Il faut enfin encore préciser que la cybernétique bénéficie, au début des années 1960, de progrès techniques importants donnant lieu à des néologismes comme, par exemple, Bionik pour décrire la construction des robots calquée sur le fonctionnement des êtres vivants. Dans le domaine du calcul automatisé, cette période correspond à l'essor de la production nationale: outre le VEB Carl Zeiss de Iéna, l'Université technique de Dresde se mit aussi à produire des calculateurs. Depuis le D1 de 1956 («D» pour Dresde), on en était arrivé à

32. Jacob Segal, «Kritische Bemerkungen zur Anwendung der Kybernetik in der Biologie », Deutsche Zeitschrift für Philosophie, 10, 1962, Heft 3, p. 324-332, ici, p. 328.

33. William Ross Ashby, Design for a Brain: the Origin of Adaptative Behaviour, Londres, Chapman and Hall, 1952.

34. William Ross Ashby, An Introduction to Cybernetics, Londres, Chapman and Hall, 1956 .

35. Kindler, 1962 , p. 3. 
une collaboration qui avait permis la réalisation du Zeiss Rechenautomat. Le physicien Nikolaus Joachim Lehmann, enlevé par les Soviétiques avec Manfred von Ardenne, était, quant à lui, revenu en $195^{8}$ et s'intéressait à la cybernétique dans le cadre de la conception des «automates à calculer».

Tout ceci concourt à l'institutionnalisation de la cybernétique en RDA, en 1961. En février, Klaus obtient du secrétaire général de l’Académie des Sciences la direction d'une «commission pour la cybernétique». C'est Thiel, alors en thèse avec Klaus, qui est secrétaire de la commission, avant que Liebscher ne le remplace. L'analyse des archives qui s'y rapportent montre néanmoins que les physiciens, tout comme les biologistes et l'administration de l'Académie, restent plutôt hostiles à la cybernétique. Lorsqu'en 1962 une «section » consacrée à cette discipline est créée, conscient du fait que celle-ci sera mieux acceptée si un mathématicien reconnu lui prête son nom, Klaus demande au mathématicien Kurt Schröder de bien vouloir en assurer la présidence ${ }^{36}$. Toutefois, la section pour la cybernétique reste paradoxalement assez isolée, et on ne trouve que très peu de contacts avec l'Institut pour les techniques de régulation.

Avant cette date, la section pour la cybernétique a surtout servi à soutenir l'organisation de plusieurs colloques, après celui organisé par la revue Einheit en avril 1961. Comme le note a posteriori avec regret Liebscher : «nous ne nous doutions vraiment pas à l'époque qu'il allait s'agir de la première et dernière conférence de ce type ${ }^{37}$ ». Quel a donc été le développement de la cybernétique après cette période?

\section{ENTHOUSIASME, «PERVERSION » ET « NORMALISATION » $(1963-1969)^{38}$}

Il est difficile d'avoir une vue schématique d'ensemble sur le développement du «point de vue cybernétique». Peut-être est-ce le recul nécessaire de l'historien qui fait défaut, les témoins de l'époque ayant aujourd'hui des opinions divergentes, parfois bien éloignées de ce que les documents d'archives pourraient

36. En novembre 1960, lors du «Symposium international sur les problèmes philosophiques de la médecine et de la biologie », au cours duquel Klaus présente un exposé sur «La signification philosophique de l'application de la cybernétique », la présence du très respecté Prof. Dr. W. Kämmerer, un des responsables de l'introduction des ordinateurs en RDA, semble jouer le même rôle de légitimation scientifique.

37. Heinz Liebscher, Fremd- oder Selbstregulation? Systemisches Denken in der DDR zwischen Wissenchaft und Ideologie, Münster, LIT Verlag, 1995, p. 20.

38. Le terme "perversion» est employé par R. Thiel dans un des entretiens qu'il nous a accordés pour décrire cette période, alors que K.-D. Wüstneck parle, quant à lui, de «période de normalisation». 
laisser présager. Il apparaît cependant que plusieurs courants se sont formés en même temps sans qu'il y ait de véritable prédominance de l'un d'eux. Le colloque d'octobre 1962, auquel participaient également des Tchécoslovaques et des Hongrois, marque sûrement un tournant décisif, «en tant que fin d'une première phase de développement de la cybernétique», écrit a posteriori

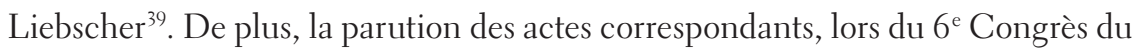
parti, obligeait les différents acteurs à modifier quelque peu leurs positions. Pour rendre compte de ces diverses évolutions, nous avons fait le choix d'un exposé assez chronologique, évitant ainsi de simplifier par nos catégories une évolution à laquelle la complexité historique est inhérente.

Janvier 1963. Walter Ulbricht est à la tribune du $6^{\mathrm{e}}$ Congrès et s'exclame: «La cybernétique est particulièrement à promouvoir ${ }^{40}$. Klaus consacre alors tout un article intitulé «La cybernétique, le programme du SED et le devoir du philosophe» au commentaire de cette déclaration ${ }^{41}$. Les cybernéticiens semblent avoir le vent en poupe et c'est alors qu'une grande réforme de l'économie est lancée avec le «nouveau système économique pour la planification et la gestion économique », mis au point par les nouveaux candidats du Politbüro, Günther Mittag et Erich Apel ${ }^{42}$. Bien plus qu'un simple plan pour l'automatisation (thème qui figure dans le discours d'Ulbricht en référence explicite à la cybernétique), le «Nouveau système économique » (NÖS) se présente comme une solution à la crise de 1960-1961, marquée par la construction du mur. Abandonnant le plan septennal en cours pour un «plan perspectif» (1964-1970), les fonctionnaires du parti appliquent les théories du Soviétique Libermann, mais aussi des théories de la régulation liées au mouvement cybernétique outre-Atlantique ${ }^{43}$. Ils

39. Les actes de la conférence sont édités en 1963: Kybernetik in Wissenschaft, Technik und Wirtschaft der DDR, Berlin, Akademie Verlag, 1963.

40. Walter Ulbricht, Das Programm des Sozialismus und die geschichtliche Aufgabe der Sozialistischen Einheitspartei Deutschlands, Berlin, Dietz Verlag, 1963, p. 345.

41. Georg Klaus, «Die Kybernetik, das Programm der SED und die Aufgaben der Philosophen ", Deutsche Zeitschrift für Philosophie, 11, 1963, p. 693-705.

42. Mittag écrira le 25 juin 1966: «Nous pensons qu'il ne suffit pas de se contenter de traduire dans la langue cybernétique les catégories économiques, mais qu'il s’agit de rendre applicable à l'économie le véritable contenu de la cybernétique: la théorie des modèles, des systèmes, de l'information, ou des boucles de régulation ", cité dans Liebscher, 1995, p. 46.

43. Le titre de la publication de Wüstneck est probablement suffisamment éloquent: «Le caractère cybernétique du nouveau système économique et la structure de modèle du plan perspectif en tant que processus cybernétique orienté vers un but», Deutsche Zeitschrift für Philosophie, 13, 1965, p. 5-31. 
s'appuient sur la prévision et l'équilibre de gestion des entreprises. Les pouvoirs de la commission de planification sont étendus et diversifiés: celle-ci devient responsable tout à la fois du plan perspectif et du plan annuel. Mais la révolution la plus profonde est celle des prix industriels, calculés désormais en fonction des coûts de production et du taux de profit planifié.

Dans un article ultérieur daté de 1993, Thiel revient sur cette période d'espoir pour les cybernéticiens, et rappelle les visées réformistes de Klaus dans le domaine économique: "Georg Klaus (...) voulait rendre l'économie plus flexible, avec Marx ${ }^{44}$.» L'implication de la cybernétique dans le NÖS allait toutefois la rendre plus que jamais dépendante des aléas politiques.

En 1964, le but de l'économie est-allemande semble être, à en croire ce qu'écrit Ulbricht, de parvenir à une «autorégulation ${ }^{45}$ ». Liebscher commence une série de sept émissions radiophoniques sur la "pensée cybernétique», alors officiellement admise. Une certaine euphorie s'empare de quelques scientifiques, proche parfois de l'exubérance. À titre d'exemple, on peut citer cet extrait d'une publication réalisée en 1965 par Klaus (alors gravement malade) et Gerda Schnauß, principalement consacrée à combattre les méfaits de la bureaucratie définie comme "collecte, transmission, traitement et enregistrement d'informations » - on retrouve ici l'importance des généralisations de la théorie de l'information - et souffrant, contrairement à la cybernétique, d'un manque d'adaptabilité. Partant du théorème d'échantillonnage de Shannon, nos deux auteurs écrivent après avoir rappelé la formule correspondante ${ }^{46}$ :

44. Rainer Thiel, «Geschlagene Truppen lernen gut?», Neues Deustchland, 25 mars 1993. Thiel avait écrit sa thèse sur les aspects cybernétiques du Capital. Il y rappelait que Marx faisait déjà la distinction entre énergie de contrôle et énergie opérationnelle, citant par exemple ce passage des Fondements de la critique de l'économie politique: «Le travail n'apparaît plus tellement comme inscrit dans un processus de production, comme si l'homme se comportait beaucoup plus comme un contrôleur ou un régulateur du processus de production.»

45. Le but est explicitement de "parvenir, sur la base du Plan et grâce à une juste utilisation des lois économiques du socialisme et à l'application du nouveau système économique pour la planification et la gestion économique, à une certaine autorégulation dans les systèmes économiques", dans Walter Ulbricht, Antwort auf aktuelle politische und ökonomische Fragen, Berlin, Deutscher Verlag, 1964.

46. Autrement dit, il faut prendre une valeur du signal toutes les « $\Delta t »$ secondes, cette quantité étant inférieure à l'inverse du double de la largeur de bande. Ce théorème est bien entendu utilisé avant tout en télécommunications. 
Le directeur du VVB [Union des entreprises du peuple] doit alors d'abord savoir quelles sont pour lui les fréquences de la fonction de signal dont il s'agit effectivement. Leur largeur de bande se laisse déterminer, et la question se pose alors de savoir avec quels écarts de temps il convient de contrôler, ordonner, etc.

Si cette théorie n'est pas appliquée, on risque soit d'y perdre en qualité (notion introduite à cette époque), soit de nécessiter un «travail administratif super$\mathrm{flu}^{47} »$. Ainsi, alors que la portée prédictive de la cybernétique était intégrée au niveau macro-économique dans les «Prognosen», cette théorie semblait pouvoir s'appliquer également à la gestion des entreprises. L'introduction des rétrocontrôles dans les schémas de fonctionnement laissait sans doute espérer plus de démocratie, comme aujourd'hui les «évaluations à $360^{\circ}$ » des gourous du management. Wüstneck insiste sur le passage de la commande (hiérarchique) à la régulation (cybernétique) et donne ainsi le schéma suivant pour les communications entre les Conseils économiques du peuple (VWR), les Groupements d'entreprises du peuple (VVB), les Entreprises du peuple (VEB) et les brigades de travail (fig. 5).

Le traité de Liebscher sorti en 1966, Kybernetik und Leitungstätigkeit (La cybernétique et les activités de direction), reçut un écho important, dont témoigne sa diffusion à vingt mille exemplaires. Dans la société cybernétique idéale, il ne s'agit plus de «diriger» comme en pays capitaliste, ni même de « contrôler » comme le veut l'économie socialiste, mais seulement de « réguler » pour accéder au communisme. Deux ans auparavant, dans un article consacré au rôle de Wiener dans le développement de la cybernétique, l'auteur avait déjà fait remarquer qu'alors que «l'anarchie de la production » gênait en régime capitaliste l'application des principes cybernétiques, ceux-ci trouvaient dans l'économie socialiste un terreau fertile.

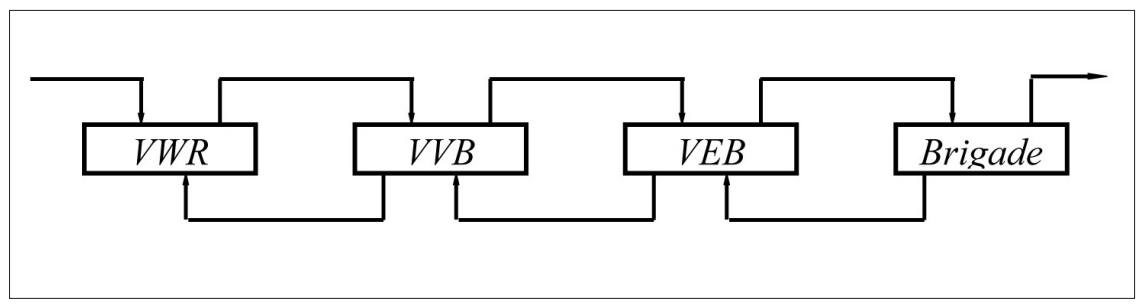

Fig. 5: D’après K.-D. Wüstneck, «Le caractère cybernétique du nouveau système économique et la structure de modèle du plan perspectif en tant que processus cybernétique orienté vers un but", Deutsche Zeitschrift für Philosophie, 13, 1965, p. 14.

47. Georg Klaus et Gerda Schnauß, « Kybernetik und sozialistische Leitung», Einheit, 20, 1965, p. 93-104, ici, p. 104 . 
En avril 1967, au $7^{\mathrm{e}}$ Congrès du parti, Ulbricht se fait plus explicite encore et annonce cette fois-ci: «Et si la cybernétique aide, alors nous nous attellerons minutieusement à cette nouvelle science jusqu'à ce que nous la maîtrisions complètement. » C'est au sujet de cette période de l'histoire de son pays que le dramaturge Heiner Müller écrit dans son autobiographie que «la cybernétique a été pendant un temps un substitut de religion pour les fonctionnaires ${ }^{48}$ ».

Selon Thiel, c'est à partir de ce moment que les «Gewis» se sentent obligés d'au moins simuler un certain intérêt pour la cause cybernétique. Wüstneck, professeur de philosophie de la Technische Hochschule Ilmenau, est élu «Kandidat» du Comité central afin d'y propager la bonne parole. Ce poste lui donne accès à tous les médias et l'introduit rapidement dans le cercle des cybernéticiens. Il participe activement à la poursuite de l'institutionnalisation de la cybernétique et se retrouve nommé directeur de la commission «cybernétique» au Secrétariat d'État pour l'enseignement technique et supérieur, chargé de mettre en place des programmes d'enseignement de la cybernétique.

Toutefois, aux dires du principal intéressé, non seulement les propositions de cette commission ne furent jamais appliquées, mais des centaines de jeunes formés à la cybernétique grâce à d'importants programmes d'échange avec l'URSS rentrèrent en RDA sans le moindre espoir de pouvoir faire valoir leur formation. La cybernétique ne fut jamais véritablement enseignée, et son sensible recul à partir de 1969 scella tristement le destin de ces apprentis cybernéticiens. Il est d'ailleurs amusant de noter qu'il semble que ce ne soit qu'à l'École du parti que la cybernétique fît parfois l'objet de cours. Des commissions ad hoc furent aussi créées au moment du $7^{\mathrm{e}}$ congrès, au ministère pour la Science et la Technique ainsi qu'au Conseil de la recherche. Cette dernière institution est apparemment la seule à avoir œuvré réellement pour la cybernétique. Wüstneck faisait partie de ces commissions officielles, mais aussi d'un "groupe de travail stratégique secret sur la cybernétique» directement sous le contrôle d'Ulbricht. Aujourd'hui très critique quant à cette institutionnalisation, Thiel qualifie ce groupe de «farce».

Cette période est en effet toujours marquée par la coexistence de travaux de valeur ${ }^{49}$. Si les élites sur le plan local entendaient parler de cybernétique,

48. Heiner Müller, Guerre sans bataille - Vie sous deux dictatures, Paris, L'Arche, 1996, p. 203. Nous remercions ici Xavier Carpentier-Tanguy pour cette indication.

49. Citons le livre de Georg Klaus et Heinz Liebscher, Was ist, Was soll Kybernetik? (Qu'est-ce que la cybernétique et que doit-elle être?), paru en 1966, qui fera l'objet de huit rééditions atteignant 100 ooo exemplaires (entre autres sous licence en République fédérale en 1970): Georg Klaus et Heinz Liebscher, Was ist, Was soll Kybernetik?, 
les directeurs d'entreprises ou autres responsables de brigades n'ont jamais pu appliquer ces théories.

Par ailleurs, le livre de Klaus sur la théorie des jeux fut l'objet d'une nouvelle série de critiques de la part de philosophes dogmatiques qui lui reprochaient ses références à des «ennemis de la classe prolétaire », comme John von Neumann, Oskar Morgenstern ou Emanuel Lasker. Ces nouvelles attaques implicitement dirigées contre la cybernétique (la théorie des jeux étant, selon Klaus, un des quatre aspects de la cybernétique, avec la théorie de l'information, des régulations et des systèmes) sont aussi sans doute liées au Printemps de Prague au cours duquel, dans plusieurs pays, nombre d'intellectuels furent accusés de «révisionnisme». C'est par un article dans Neues Deutschland, le 30 avril 1969, que Liebscher est personnellement mis en cause. Son détracteur, Kurt Hager, célèbre idéologue du parti, se réfère aux discussions de la veille, lors du $10^{\mathrm{e}}$ Plenum du Comité central du parti, où un article publié par Liebscher dans Spektrum avait été critiqué. Il devait d'ailleurs s'agir d'autre chose que d'une simple «critique», puisque le rédacteur en chef de la revue fut, entre autres, immédiatement suspendu de ses fonctions. En quelques mots, disons que c'est surtout une éventuelle remise en cause du rôle directeur du parti qui crée la panique au sein du Comité central ${ }^{50}$.

Si la réunion d'avril 1969 marque un virage anticybernétique très clair, la

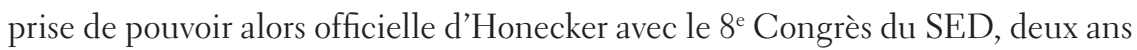
plus tard, ne laisse plus d'espoir à cette discipline: le nouveau secrétaire général annonce fièrement: «Il est maintenant enfin établi que la cybernétique et la recherche systémique ne sont que des pseudo-sciences ${ }^{51}$.» Toutefois, là encore,

Leipzig, Jena et Berlin, Urania Verlag, 1966 ( $9^{\mathrm{e}}$ éd. 1974); La théorie des jeux d'un point de vue philosophique, de Klaus, qui paraît deux ans plus tard: Georg Klaus, Spieltheorie in philosophischer Sicht, Berlin, Deutscher Verlag der Wissenschaften, 1968; et des livres qui ne connaîtront pas la même postérité, tels que La cybernétique dans le combat contre la criminalité, traduction de quelques articles soviétiques sur ce thème: Die Kybernetik im Kampf gegen die Kriminalität, Potsdam, Institut Theorie der Sozialistischen Staatlichen Führung, 1967.

50. Un texte de 1966 de Liebscher, commandé par le Politbüro dans le but d'étudier les possibilités de réorganisation du système d'information du parti, lui avait déjà valu des remontrances exprimées par Hager, Honecker et Mittag. Les remarques qu’ils suggéraient alors se fondaient exclusivement sur une interprétation cybernétique de la notion d'information, ce qui ne correspondait pas aux attentes du Politbüro. Liebscher, 1995, p. 72-75.

51. Là encore, c'est en suivant les articles dans Einheit que l'on peut comprendre la position officielle du parti. Hager y écrit dans le numéro de novembre 1971: "Quelle que soit l'importance de la cybernétique et de la théorie des systèmes, nous ne pouvons 
la situation est plus complexe qu'elle n'en a l'air. Si l'on peut presque considérer l'avant-propos de Klaus à la troisième édition, en 1973, de son livre Cybernétique et société comme une autocritique, il n'en continue pas moins de publier sur la cybernétique, mais dans des collections comme «critique de l'idéologie bourgeoise » où paraît, en 1973, Kybernetik - eine neue Universalphilosophie der Gesellschaft? (Cybernétique, science universelle de la société?).

L'abandon du nouveau système économique cause, bien sûr, du tort à la cybernétique dont elle constituait pour ainsi dire les fondements scientifiques. À partir des années 1970, on ne parle plus de cybernétique mais de microélectronique ou d'informatique. Il semblerait curieusement que ce ne soit qu'en économie que la cybernétique ait pu conserver son droit de cité, le dernier colloque sur ce thème en économie ayant eu lieu en 1985. Pour le reste, la cybernétique semble avoir représenté trop de remises en cause pour le régime.

Que tirer de l'analyse de ces rapports pour le moins «dialectiques » entre cybernétique et matérialisme dialectique? Une remarque d'épistémologie ou de sociologie des sciences se dégage d'abord sur la façon dont se clôt une controverse. L'ensemble de cette étude ne constitue en fait que l'analyse d'une polémique autour de la validité des théories cybernétiques. Au départ, lorsque la cybernétique était marquée du sceau de "science bourgeoise », la controverse se retrouvait reléguée dans des modes de discours officieux, aujourd'hui seulement accessibles par les techniques de l'histoire orale. Les documents d'archives, profondément marqués par leur mode et leur contexte de production, peuvent biaiser l'interprétation qu'en fait l'historien. Ce n'est que grâce aux entretiens qu'il devient possible de comprendre comment telle ou telle lettre, apparemment banale, peut avoir une répercussion importante, par exemple, sur l'institutionnalisation de la cybernétique. D'un autre côté, les témoins parlent après la résolution des tensions liées à la controverse, même si cette histoire comporte encore une dimension d'actualité. Régulièrement, le pouvoir politique intervenait pour tenter de clore la controverse. Ceci est surtout visible dans les formes bien établies du discours et les allocutions aux différents congrès sont souvent emblématiques: aux $6^{\mathrm{e}}$ et $7^{\mathrm{e}}$ congrès, l'intervention se fait en faveur de la cybernétique, avec constitution d'une histoire officielle la créditant des progrès

tolérer qu'elles prennent la place du matérialisme historique dialectique, de l'économie politique du socialisme, du communisme scientifique ou de la science socialiste de la direction; qu'elles deviennent la science absolue et que le langage d'une science spécifique devienne la langue du Parti. Le Parti cesserait alors d'être un parti marxiste-léniniste », Einheit, 26, 1971, p. 1203-1242, ici p. 1215. 
de l'aéronautique ou de l'informatique; et au congrès suivant, il s'agit de la classer définitivement parmi les pseudo-sciences. On est, de plus, en présence d'un rapport souvent inversé entre les sources disponibles et les points de vue représentés : lors de l'établissement progressif de la cybernétique, avant son acceptation officielle, on ne retrouve que les positions anti-cybernétiques du parti; par la suite, pour l'ère Ulbricht, on trouve peu de traces des «Gewis » qui y étaient hostiles. Alors que la cybernétique a été plus ou moins rejetée par le régime de Honecker, les témoins que nous avons rencontrés se présentent aujourd'hui, à un degré ou un autre, comme des cybernéticiens victimes de la disparition, en 1990, de la RDA après son adhésion à la RFA.

Finalement, au regard de l'histoire politique, il est opportun, vingt ans après la chute du mur de Berlin, de s'interroger sur l'importance des courants réformateurs dans le développement de la cybernétique. La majorité des défenseurs de la cybernétique en RDA ont été des intellectuels critiques du régime, voire des dissidents. Rappelons, à titre d'exemple, la présence de Robert Havemann, dissident notoire à partir de 1963, lors de la première manifestation cybernétique est-allemande, la journée d'information organisée par la revue Einheit deux ans plus tôt. Il serait toutefois simpliste d'affirmer que les « cybernéticiens » étaient, sinon réformateurs, du moins progressistes, puisque beaucoup des témoins interrogés aujourd'hui se définissent volontiers comme ayant été toujours critiques face au parti ou à la Stasi, positions grandement favorisées à l'époque actuelle. Cependant, la remise en cause du rôle dirigeant du parti et la revendication d'une structure plus souple, à l'écoute de la base, dans le modèle de planification et de décision constituaient à n'en pas douter une volonté de réformer le pays, sans pour autant abandonner le marxisme.

En ce qui concerne la planification économique, une citation de Thiel tirée de Neues Deutschland mérite d’être rappelée: «Georg Klaus (...) voulait rendre l'économie plus flexible. Avec Marx ${ }^{52}$.» Cet article, paru le 25 mars 1993, a suscité une vive polémique dans la rédaction du journal autour de la thèse selon laquelle la RDA aurait pu être «sauvée » grâce à la cybernétique. La caricature de 1967 dans l'unique hebdomadaire satirique de la RDA a récemment été reprise dans un ouvrage collectif sur la contamination de l'Est par la cybernétique (fig. 6).

C'est lorsqu'elle a commencé à mettre en cause le régime autoritaire, préconisant des fonctionnements plus démocratiques, que la cybernétique a été condamnée. Des concepts importants, comme celui de flexibilité, introduit

52. Rainer Thiel, «Geschlagene Truppen lernen gut?», Neues Deustchland, 25 mars 1993 , P. 7 . 


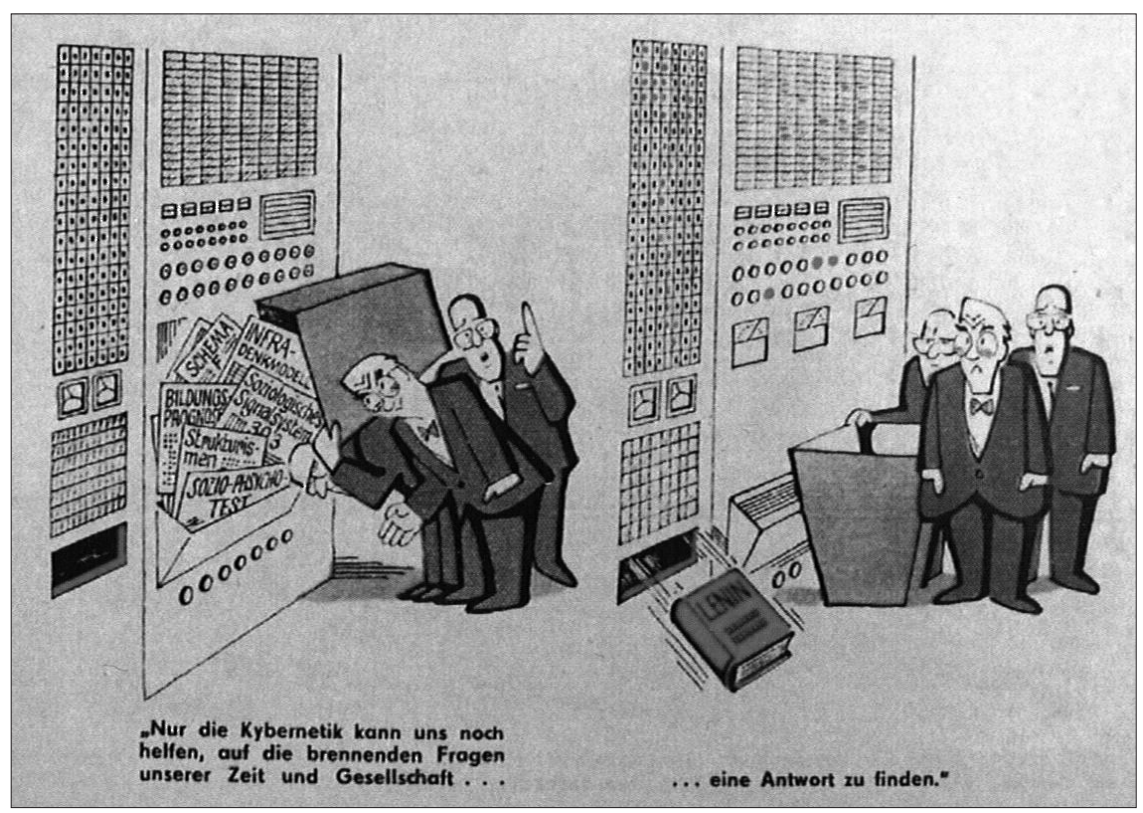

Fig. 6: Frank Dittmann et Rudolf Seising, Kybernetik steckt den Osten an. Aufstieg und Schwierigkeiten einer interdisziplinären Wissenschaft in der DDR, Berlin, trafo, 2007, p. 16. Traduction: «Face aux questions brûlantes de notre temps, seule la cybernétique peut encore nous aider... à trouver une réponse. »

dans les années 1960, ont aujourd'hui été détournés aux dépens des travailleurs, soumis à toujours plus de précarité. Pourtant, la crise récente et persistante de l'économie capitaliste est devenue structurelle. Aujourd'hui, les entrepreneurs que l'on distingue sur cette caricature seraient tout aussi perplexes que leurs congénères de la fin des années 1960, en observant que quelles que soient les informations entrées dans la machine, la bible qui en sortirait ne serait plus un ouvrage de Lénine mais les marges nécessaires de profit des grands groupes. D'une certaine façon, l'histoire de la cybernétique en RDA nous amène à conclure que pour diriger une société de façon juste, il faut d'abord s'entendre sur les valeurs à défendre avant de pouvoir programmer ensemble. 